Artículo científico

Volumen 31(2):367-383. Mayo-agosto, 2020

e-ISSN 2215-3608, doi:10.15517/am.v31i2.34894

http://www.revistas.ucr.ac.cr/index.php/agromeso

\title{
Análisis de la estructura del sector y la asociación público-privada de semillas de maíz en México ${ }^{1}$
}

\section{Analysis of the sector structure and the public-private partnership of maize seed in Mexico}

\author{
M. Laura Donnet ${ }^{2}$, Iraís Dámaris López-Becerril ${ }^{3}$, Ciro Dominguez ${ }^{4}$, Juan Arista-Cortés ${ }^{5}$
}

1 Recepción: 14 de julio, 2019. Aceptación: 5 de diciembre, 2019. Este trabajo formó parte de la Investigación del Programa de Socioeconómica del Centro Internacional de Mejoramiento de Maíz y Trigo (CIMMYT) para la iniciativa Modernización Sustent able de la Agricultura Tradicional (MasAgro) de México, durante enero de 2011 a enero de 2017.

2 Economista agrícola independiente. 3000 Santa Fe, Argentina. lauradon105@hotmail.com (https://orcid.org/0000-0002-4932-0561).

3 Estadística independiente. Texcoco, Edo. de México, México. CP 56237. idamarislb@hotmail.com (https://orcid.org/0000-0002-9358-4994),

4 School of Agriculture, Policy and Development, University of Reading, RG6 6AR, Berkshire, Reading, Inglaterra. c.dominguezmendez@ pgr.reading.ac.uk (https://orcid.org/0000-0003-0994-8204).

5 Centro Internacional de Mejoramiento de Maíz y Trigo (CIMMYT), Texcoco, Edo. de México, México.CP 56237.j.arista@ cgiar.org (https:// orcid.org/0000-0003-4015-797X).

\section{Resumen}

Introducción. El rápido crecimiento de la inversión privada en el sector de semillas de maíz plantea desafíos para los organismos públicos y las empresas más pequeñas que tienen cada vez menor acceso a tecnologías y mercados. El Consorcio Internacional de Mejoramiento de Maíz (IMIC-LA), creado en México en 2011, busca aumentar el tamaño y la competitividad del sector semillero mediante el acceso a germoplasma y herramientas de mejoramiento. Sin embargo, no hay datos y medidas básicas recientes de la estructura que permitan evaluar esta y otras iniciativas del sector. Objetivo. Actualizar y analizar los indicadores de ventas totales, participación en ventas por categorías de empresas y tipos de variedades de semilla, y cobertura con semillas mejoradas, en México. Materiales y métodos. Se utilizaron datos de ventas de semillas de maíz de entrevistas y encuestas con empresas semilleras y estadísticas oficiales de producción de semillas del Servicio Nacional de Inspección y Certificación de Semillas (SNICS) del año 2011 al año 2016. Resultados. Los resultados mostraron un sector semillero más grande y competitivo después de veinticinco años de apertura, especialmente en los años más recientes en los que se destaca el rápido aumento del volumen y la participación de las ventas del subsector de empresas nacionales. Conclusión. La amplia participación de las empresas como miembros del consorcio y el desarrollo y comercialización de nuevas variedades, sugieren un impacto positivo y un papel en el crecimiento y competitividad de las empresas semilleras nacionales.

Palabras clave: producción de semillas, alianzas, industria de semillas, mercados, producción de semillas híbridas.

Abstract

Introduction. The rapid growth of private investment in the maize seed sector poses challenges for public breeding organizations and smaller seed companies that have increasingly less access to technologies and markets. The International 


\begin{abstract}
Maize Improvement Consortium (IMIC-LA) created in Mexico in 2011, seeks to increase the size and competitiveness of the seed sector through access to germplasm and breeding tools. However, there are no recent basic data and measures of the sector structure that allow evaluating this and other sector initiatives. Objective. To update and analyze the structure indicators of total seed sales, participation in sales by categories of companies and type of seed varieties, and coverage with improved seeds in Mexico. Materials and methods. Maize seed sales data from interviews and surveys with seed companies and official seed production statistics from the Servicio Nacional de Inspeccion y Certificacion de Semillas (SNICS) from 2011 to 2016 were used. Results. The results showed a larger and more competitive seed sector after twenty-five years of opening, especially in the most recent years in which the rapid increase in volume and the share of sales of the national business subsector stands out. Conclusion. The broad participation of seed companies as members of the consortium and the development and commercialization of new seed varieties suggest a positive impact and a role of the consortium in the growth and competitiveness of the national subsector.
\end{abstract}

Keywords: seed production, alliances, seed industry, markets, hybrid seed production.

\title{
Introducción
}

El sector semillero es el conjunto de organizaciones que participan en el mejoramiento, producción y distribución de semillas y brindan a los agricultores las innovaciones de mejoramiento genético a través de semillas comerciales. Este sector es parte de un sistema de semillas más amplio que incluye la selección, siembra e intercambio por parte de los agricultores. En el caso del maíz, en los países con agricultura desarrollada, prácticamente todos los productores utilizan las semillas comerciales, principalmente híbridos de maíz, mientras que en los países con economías agrícolas menos desarrolladas la adopción de híbridos está creciendo rápidamente, lo que estimula el desarrollo de la industria privada de semillas (Fischer et al., 2014). Por ejemplo, en la India (Pray y Nagarajan, 2012; 2013), Tailandia (Napasintuwong, 2014), y países de África del sur (Chisinga, 2011; Hamukwala et al., 2012; Mabaya, 2016), las empresas privadas están creciendo a partir de un sector previamente desarrollado y dominado por empresas estatales. En Ghana y Uganda, el crecimiento se está dando a partir de una producción comercial de semillas muy escasa, dominada hasta hace poco por productores de muy pequeña escala (Tripp y Mensah-Bonsu, 2013; Tripp y Ragasa, 2015). En Kenia (Smale y Olwande, 2014), Etiopía (Abate et al., 2015; Sisay et al., 2017) y la mayoría de los países de África occidental (Fischer et al., 2014), las empresas privadas están creciendo rápidamente aunque, el estado continúa dominando el sector.

En México, en 1991 se permitió la participación del sector privado en la investigación, producción y comercialización de semillas. Previamente el sector había tenido un desarrollo dominado por el sector público, con el Instituto Nacional de Investigaciones Agrícolas, Forestales y Pecuarias (INIFAP), en el mejoramiento y desarrollo, y la Productora Nacional de Semillas (PRONASE) en la producción y distribución. Tras la apertura, se produjo una rápida entrada de empresas semilleras multinacionales y un cierre paulatino de la PRONASE (Luna et al., 2012; Espinosa et al., 2014). El sector público continuó su participación en investigación y mejoramiento de maíz, principalmente a través del INIFAP, aunque con recursos cada vez más restringidos (Espinosa et al., 2014).

El crecimiento de la inversión privada y disminución de la capacidad y contribución de la investigación pública en el mejoramiento, producción y comercialización de semillas (Langyintuo et al., 2010; Beintema et al., 2012; Spielman et al., 2012; Flaherty et al., 2013), propone desafíos para los organismos públicos y las empresas más pequeñas, que tienen cada vez menor participación en la generación y acceso a la tecnología y los mercados. Para las empresas se plantea la necesidad de acceder al germoplasma y los avances en mejoramiento para mantenerse competitivas (Langyintuo et al., 2010; Schenkelaars et al., 2011; Kassie et al., 2013; Ragonnaud, 2013). Para 
los centros públicos de investigación, se expone el desafío de colaborar con el sector privado para llegar a los productores y generar impacto con los productos de su investigación (Spielman et al., 2007).

En México, los centros de mejoramiento público manifestaron la necesidad de desarrollar nuevos esquemas de abasto de semilla básica con las empresas, para que sus productos de mejoramiento se comercialicen con los agricultores (Espinosa et al., 2003; González et al., 2008). Las empresas semilleras, por su parte, plantearon la necesidad de acceder a un abastecimiento de germoplasma adecuado y oportuno que asegure una producción de semilla comercial viable (Luna et al., 2012; Castañeda et al., 2014). Del lado de los centros de investigación agrícola internacionales, como el Centro Internacional de Mejoramiento de Maíz y Trigo (CIMMYT), y que en el pasado tendían a informar el impacto por la cantidad de tecnologías desarrolladas, se planteó el desafío de demostrar el impacto de los productos de investigación, incluidas las semillas mejoradas, en los campos de los agricultores (Hellin y Camacho, 2017).

En 2011, se creó en México el Consorcio Internacional de Mejoramiento de Maíz para Latinoamérica (IMIC-LA), dentro del programa de Modernización Sustentable de la Agricultura Tradicional (MasAgro) entre el CIMMYT, centros nacionales de investigación y mejoramiento genético, y empresas semilleras nacionales. El objetivo del consorcio es mejorar la productividad del maíz y aumentar el tamaño y la competitividad del sector semillero en México, mediante el acceso selectivo a germoplasma y herramientas de mejoramiento y el fortalecimiento de la capacidad de las instituciones locales (Prasanna, 2013). El IMIC-LA se enfoca en la adopción de semillas mejoradas de maíz por parte de agricultores con potencial para aumentar la productividad especialmente en zonas de producción de temporal en México (García y Ramírez, 2014; Escandón et al., 2017). En México, IMICLA es conocido como MasAgro Maíz.

Un estudio reciente señala que las empresas semilleras mexicanas recibieron un apoyo significativo a partir de la posibilidad de acceder a nuevo germoplasma mejorado disponible con el consorcio de semillas de MasAgro (Castañeda et al., 2014). Sin embargo, si no se conocen medidas básicas de la estructura como el tamaño de ventas totales y la participación o proporción de las ventas de las distintas empresas, no se pueden evaluar políticas y decisiones de gestión. Conocer las medidas de la estructura del sector es fundamental para evaluar con precisión las opciones de política para diseñar estrategias nacionales de crecimiento agrícola, establecer prioridades de investigación pública y diseñar incentivos privados para la innovación y para la adopción de tecnologías que mejoren la productividad (Spielman y Kennedy, 2016). Para el sector de semillas de maíz en México, los datos más recientes del tamaño del sector son de 2009 (Luna et al., 2012) y los datos más completos con participación de mercado de empresas y variedades de semilla son de 1993 y 1995 (López, 1995; Morris y López, 1999), con lo cual falta información y datos más actualizados que permitan una mayor comprensión y dimensionamiento de cómo funciona el sector y las perspectivas de una alianza público-privada entre las empresas semilleras y los centros de investigación para aumentar el tamaño y mejorar la competitividad.

Por su importancia en México, la semilla de maíz está en el centro de debates y discusiones de política sobre qué tipos de semillas y sistema de producción asociados es el mejor (Turrent et al. 2017), cuáles son niveles adecuados de participación de los materiales de mejoramiento público y de empresas nacionales y multinacionales (Espinosa et al., 2014; Luna et al., 2012). La intención de presentar y analizar nuevos datos y evidencia sobre la estructura, es contribuir a evaluar y mejorar la gestión del consorcio y de otras políticas y a enriquecer el debate público sobre las opciones de semillas para mejorar la producción de maíz en México.

El objetivo de este trabajo fue actualizar la información y analizar los indicadores de ventas totales, participación en ventas por categorías de empresas, tipos de variedades de semilla, y cobertura con semillas mejoradas en México. El análisis se centró en los cambios con respecto a años anteriores y con la participación del nuevo consorcio de semillas IMIC-LA. 


\section{Materiales y métodos}

Este estudio se basó principalmente en datos de ventas de semillas de maíz recolectados a través de entrevistas y encuestas realizadas por el Centro Internacional de Mejoramiento de Maíz y Trigo (CIMMYT), con las empresas semilleras participantes en el consorcio IMIC-LA durante los años 2011 a 2016. En 2011 y 2012 se entrevistaron 31 empresas semilleras (entrevistas con empresas semilleras CIMMYT - MasAgro, 2011 y 2012). De 2013 a 2016 se encuestaron, vía correo electrónico, 30, 24, 39 y 41 empresas en 2013, 2014, 2015 y 2016, respectivamente (encuestas de comercialización de semillas CIMMYT - MasAgro, 2013, 2014, 2015 y 2016). Con las entrevistas se obtuvieron datos de ventas globales de semilla de maíz de las empresas por año. Con las encuestas se obtuvieron datos de ventas de semillas de maíz por tipos de variedades.

A partir de estas entrevistas y encuestas se obtuvieron datos de 47 empresas semilleras nacionales. Estos datos no estaban completos para todas las empresas todos los años de 2011 a 2016. Los datos faltantes se estimaron con base en el crecimiento promedio del conjunto de empresas con datos u observaciones cada año por categorías de tamaño.

La clasificación de empresas según el tamaño se basó en la definición de MacRobert (2014) de empresas pequeñas como aquellas que tienen menos de 500 toneladas de producción o ventas de semilla de maíz por año. El resto de las categorías se definieron en relación con la definición anterior: hasta diez veces más grande (5005000 toneladas) se consideraron empresas medianas, más de diez veces más grandes empresas grandes ( $>5000$ toneladas), y menos de diez veces más pequeñas ( $<50$ toneladas), se consideraron microempresas.

Los datos de 47 empresas, observados y estimados, se complementaron con datos estimados de las ventas de empresas multinacionales y nacionales, que no participan en el consorcio IMIC-LA, a partir de estadísticas de producción de semilla certificada de maíz ciclos 2011/2016 del SNICS (2016). La estimación se realizó tomando los datos de producción reportados por SNICS como si fueran datos de ventas, es decir, equivalentes a los datos reportados y estimados a partir de las entrevistas y encuestas. Combinando los datos de ventas de las entrevistas y encuestas, y los de producción de semilla de SNICS, se obtuvieron datos estimados completos del sector para los años 2011 a 2016. Los datos de ventas de las encuestas y de producción del SNICS, se sumaron para el análisis en este estudio.

Para el análisis de los tipos de variedades de semilla se utilizó información de la encuesta de impacto del germoplasma CIMMYT-MasAgro (2015), que se realizó con las empresas participantes en el consorcio de IMIC-LA, vía correo electrónico, para conocer el origen y uso del germoplasma de maíz. Esta información se utilizó para clasificar los híbridos y variedades (de polinización abierta) de maíz en públicos, privados, CIMMYT (germoplasma) con IMIC-LA, públicos (germoplasma) con IMIC-LA y privados (germoplasma) con IMIC-LA, en función del autor de la variedad o cruza final y de las líneas y cruzas simples componentes de los híbridos. Con base en esta información, se clasificaron las variedades de semillas vendidas en 2016 por las empresas del consorcio según la encuesta de comercialización de semillas CIMMYT - MasAgro, 2016.

Todos los datos se trataron manteniendo la confidencialidad de la información de las empresas y los resultados del análisis se presentaron como promedios y categorías por tipos de empresas y productos.

Para la estimación de uso y mercados de semilla, se utilizaron datos de estadísticas de producción de maíz y estadísticas de uso tecnológico del Servicio de Información Agrícola y Pecuaria (SIAP), y las zonas de producción de maíz de temporal (Donnet et al., 2017).

Los datos de la estructura del sector para los años 2011 a 2016, se compararon con datos disponibles de tres publicaciones anteriores de 1995, 1999 y 2012 (Cuadro 1). Las publicaciones de 1995 y 1999 reportaron datos de encuestas con empresas semilleras realizadas por el CIMMYT en 1993 y 1996, respectivamente; mientras que la publicación de 2012, utilizó datos de producción de semillas en 2009 de SNICS. Las tres publicaciones reportaron tamaño del sector de semillas de maíz, participación del sector público y del sector privado, y el número de 
Cuadro 1. Datos de la estructura del sector semillero de maíz en México utilizados para el análisis en este trabajo.

Table 1. Data on the structure of the maize seed sector in Mexico used for the analysis in this study.

\begin{tabular}{|c|c|c|c|}
\hline & México, 1993 & México, 1996 & México, 2009 \\
\hline Publicación & López (1995) & Morris y López (1999) & Luna et al. (2012) \\
\hline Fuente de los datos & $\begin{array}{l}\text { CIMMYT, encuesta de hechos y } \\
\text { tendencias mundiales 1993/1994 }\end{array}$ & $\begin{array}{l}\text { CIMMYT, encuesta de } \\
\text { impacto mejoramiento }\end{array}$ & SNICS \\
\hline $\begin{array}{l}\text { Tamaño del sector (toneladas de semilla } \\
\text { de maíz) }\end{array}$ & 42460 & 32600 & 45000 \\
\hline Número de empresas multinacionales & 5 & 5 & * \\
\hline Número de empresas nacionales & 13 & 50 & $*$ \\
\hline Número de productores de semilla & 37 & 7 & $*$ \\
\hline $\begin{array}{l}\text { Participación de mercado de sector } \\
\text { público }(\%)\end{array}$ & 9 & 13 & 0 \\
\hline $\begin{array}{l}\text { Participación de mercado de sector } \\
\text { privado - total }(\%)\end{array}$ & 91 & 87 & 100 \\
\hline Empresas nacionales (\%) & $*$ & 17 & 5 \\
\hline Empresas multinacionales (\%) & $*$ & 70 & 95 \\
\hline Híbridos privados & 62 & $*$ & $*$ \\
\hline Híbridos públicos & 31 & $*$ & $*$ \\
\hline Variedades de polinización libre & 7 & $*$ & * \\
\hline Área de maíz (1000 ha) & 7348 & 7900 & 8000 \\
\hline Superficie sembrada con híbridos (\%) & 26 & 19 & $*$ \\
\hline $\begin{array}{l}\text { Superficie sembrada con variedades } \\
\text { mejoradas }(\%)\end{array}$ & 10 & 1 & $*$ \\
\hline $\begin{array}{l}\text { Superficie sembrada con semilla del } \\
\text { productor }(\%)\end{array}$ & 64 & 80 & $*$ \\
\hline
\end{tabular}

* sin información / * whithout information.

hectáreas plantadas con maíz. Las publicaciones basadas en las encuestas, adicionalmente, reportaron el número de empresas nacionales, multinacionales y de productores de semilla, y el porcentaje de la superficie de maíz plantada con híbridos, con variedades mejoradas y con semilla del productor. Las publicaciones de 1999 y 2012 tienen información de la participación de mercado de las empresas nacionales y empresas multinacionales, y la publicación de 1995 tiene información acerca de la participación de mercado de híbridos privados e híbridos públicos.

Estos indicadores se consideraron puntos de comparación relevantes de la estructura del sector al inicio de la apertura comercial en los 90 y antes de iniciar el consorcio de semillas IMIC-LA. Los resultados presentan los mismos indicadores calculados con los nuevos datos en este estudio.

\section{Resultados}

\section{Participantes y formas de coordinación}

El sector de semillas de maíz en México está integrado por empresas semilleras y centros públicos de investigación y mejoramiento nacionales y el CIMMYT (Figura 1). El sector semillero se puede ver como un 


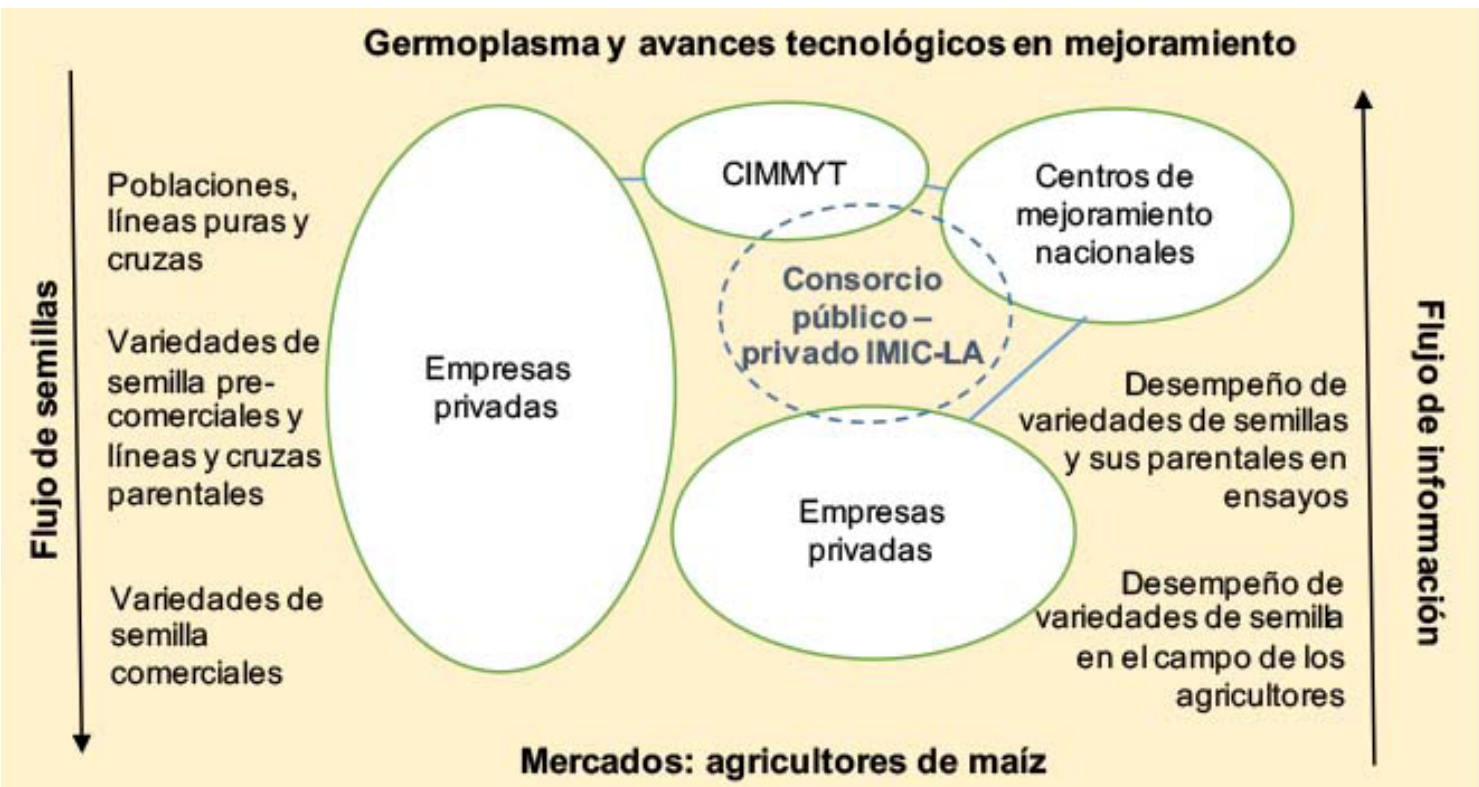

Figura 1. Sector semillero de maíz en México. 2011 - 2016.

Figure 1. Maize seed sector in Mexico. 2011 - 2016.

sistema que mueve tanto productos de semillas como información sobre su desempeño entre los recursos de germoplasma y avances en mejoramiento, y los agricultores (Christensen y Nelson, comunicación personal, 2012). La coordinación de este sistema se realiza de tres formas. Una forma es la integración del mejoramiento, producción y comercialización en una misma empresa; este es el caso de las empresas que tienen programas de mejoramiento y, desarrollan y registran variedades de semillas privadas o propietarias.

Otra forma de coordinación es la multiplicación por parte de empresas semilleras de los híbridos y variedades públicos desarrollados por los centros de investigación nacionales. Esta forma es utilizada por las empresas que no tienen programas de mejoramiento, aunque también por empresas que, teniendo variedades de semillas privadas, multiplican otras de origen público. La coordinación se realiza a través del abastecimiento de semilla básica de la variedad, en el caso de variedades de polinización libre, y de las líneas y cruzas simples progenitores en el caso de híbridos.

Con el consorcio IMIC-LA, hay una nueva forma de coordinación que es la alianza público-privada integrado por el CIMMYT, centros de mejoramiento nacionales, incluyendo el INIFAP y universidades nacionales, y empresas semilleras nacionales miembros o socias del consorcio. El CIMMYT y los centros de mejoramiento nacionales participan en el mejoramiento y desarrollo de variedades de semilla de uso final. La asociación en el consorcio público-privado, involucra un conjunto de actividades y compromisos propios que los socios realizan tanto individualmente como en conjunto. El CIMMYT desarrolla productos de mejoramiento que incluye cruzas de híbridos para ser multiplicadas como productos de semillas finales y cruzas simples, y líneas para ser usadas como productos intermedios de mejoramiento por las empresas y centros de mejoramiento en el consorcio. La producción de híbridos finales es una nueva actividad del CIMMYT con el consorcio de IMIC-LA, mientras que el desarrollo de productos intermedios, líneas y cruzas simples, con las que las empresas y los centros de mejoramiento nacionales desarrollaban los híbridos finales, es la actividad que el CIMMYT venía desarrollando regularmente.

Los socios del consorcio IMIC-LA participan en una red colaborativa de evaluación que consiste en ensayos multilocales en los que se evalúan los productos de mejoramiento. Los miembros del consorcio deben aportar una 
localidad de evaluación como mínimo, con lo cual tienen derecho a incluir una variedad de semilla producto de su propio mejoramiento para su evaluación. La red de evaluación incluye ensayos de mejoramiento participativo de maíces nativos. El CIMMYT coordina los procesos de recepción y preparación de las variedades de semilla que se quieren probar, diseño experimental y envío de los materiales a las localidades de evaluación. Las empresas participantes del consorcio, reciben las semillas para un ensayo en el sitio de evaluación acordado y se encargan de la conducción del ensayo, incluyendo la siembra, manejo agronómico, cosecha y toma de datos. El CIMMYT recopila, analiza y distribuye la información sobre el rendimiento y otras características de los productos de mejoramiento. Esta información es utilizada por las empresas para identificar híbridos finales que se desean multiplicar; adicionalmente, se prueban en los ensayos productos intermedios de mejoramiento, líneas y cruzas simples de maíz, que se evalúan por sus características como progenitores de cruzas finales. La información a partir de los ensayos de progenitores es utilizada por las empresas y los centros de investigación públicos miembros del consorcio interesados en el mejoramiento; el cual está enfocado en el desarrollo de híbridos de alto rendimiento y resistentes al estrés en las condiciones de producción de temporal en los distintos mega-ambientes tropical, subtropical y de valles altos de México.

Las actividades del consorcio incluyen investigación, desarrollo y capacitación en tecnología de producción de semillas y en tecnologías de mejoramiento, por ejemplo dobles haploides. También se realizan actividades de promoción de las semillas mejoradas y tecnologías asociadas de producción de maíz en parcelas demostrativas con los agricultores.

\section{Empresas, tamaño y participación de mercado}

En el 2016 el sector estuvo integrado por 114 empresas productoras de semilla, dos empresas grandes multinacionales, 14 empresas medianas (una multinacional y el resto nacionales), 53 empresas pequeñas (una multinacional y el resto nacionales) y 45 microempresas nacionales (Cuadro 2). Con respecto a los datos de la estructura disponibles previamente (Cuadro 1), se observó un aumento en el número total de empresas de prácticamente el doble, de 55 empresas en 1993 y 62 en 1996, a 114 en 2016. Durante los años cubiertos por este estudio, se observaron cambios

Cuadro 2. Empresas semilleras de maíz en México. 2011 - 2016.

Table 2. Maize seed companies in Mexico. 2011 - 2016.

\begin{tabular}{|c|c|c|c|c|c|c|c|}
\hline & & 2011 & 2012 & 2013 & 2014 & 2015 & 2016 \\
\hline \multirow[t]{4}{*}{ Multinacionales } & Grandes & 1 & 1 & 2 & 1 & 1 & 2 \\
\hline & Medianas & 1 & 2 & 0 & 3 & 3 & 1 \\
\hline & Pequeñas & 0 & 0 & 1 & 0 & 0 & 1 \\
\hline & Micro & 1 & 0 & 0 & 0 & 0 & 0 \\
\hline \multirow[t]{3}{*}{ Nacionales } & Medianas & 8 & 9 & 10 & 10 & 14 & 13 \\
\hline & Pequeñas & 42 & 34 & 55 & 40 & 43 & 52 \\
\hline & Micro & 52 & 52 & 75 & 44 & 42 & 45 \\
\hline Total & & 105 & 98 & 143 & 98 & 103 & 114 \\
\hline
\end{tabular}

Fuente: Datos de entrevistas y encuestas con empresas semilleras del consorcio MasAgro y de producción de semilla certificada del SNICS. Empresas grandes: $>5000$ t de semilla de maíz, medianas: 5000-500 t; pequeñas 500-50 t; micro <50 t / Source: Data from interviews and surveys of seed companies in the MasAgro consortium and certified seed production statistics of SNICS. Large companies : $>5,000 \mathrm{t}$ of maize seed, medium: 5,000-500 t; small 500-50 t; micro $<50 \mathrm{t}$. 
en el número de empresas en cada categoría, resaltándose el aumento sostenido del número de empresas medianas del subsector nacional, indicando el crecimiento del tamaño de las empresas en este subsector. La participación en el consorcio de MasAgro fue de aproximadamente cincuenta empresas nacionales en 2016.

Los cambios en el tamaño y participación de mercado del sector semillero de maíz en México en los seis años cubiertos por este estudio del 2011 al 2016, se muestran en la Figura 2. El tamaño varió entre un mínimo de 53000 t en el 2012 y un máximo de más de 101000 t en 2015. El tamaño fue de 85263 t en 2016, último año cubierto por este estudio, con un crecimiento del $35 \%$ y una tasa promedio de crecimiento del 6,1\% entre 2011 y 2016. Este tamaño representa un aumento de tamaño del doble con respecto a 1993, casi el triple de la cifra disponible para 1996, y el doble de la cifra reportada para 2009, lo que indica el rápido crecimiento del sector en los últimos siete años. La participación en ventas de los subsectores varió entre un máximo de $76 \%$ en 2011 y un mínimo de $69 \%$ en 2016 para las empresas multinacionales y, en sentido opuesto, un mínimo de $24 \%$ y un máximo de $31 \%$ para las nacionales en 2011 y 2016, respectivamente. La participación en ventas de empresas nacionales del $31 \%$ en 2016 fue similar a la registrada en 1996, con la diferencia de que, en este año, el $30 \%$ de participación nacional correspondía un $13 \%$ al sector público y un $17 \%$ al sector privado (Cuadro 1). Por el contrario, hubo un notable aumento con respecto al $5 \%$ de participación reportada para las empresas nacionales en 2009. La clasificación por tamaños mostró que en el año 2016 la categoría con mayor participación fue multinacionales grandes con el 68 $\%$, seguida por las nacionales medianas con el $20 \%$, multinacionales medianas y las nacionales pequeñas con el $10 \%$ cada una. Además, hubo un $1 \%$ de participación de una empresa multinacional mediana y otro $1 \%$ de las empresas nacionales micro.

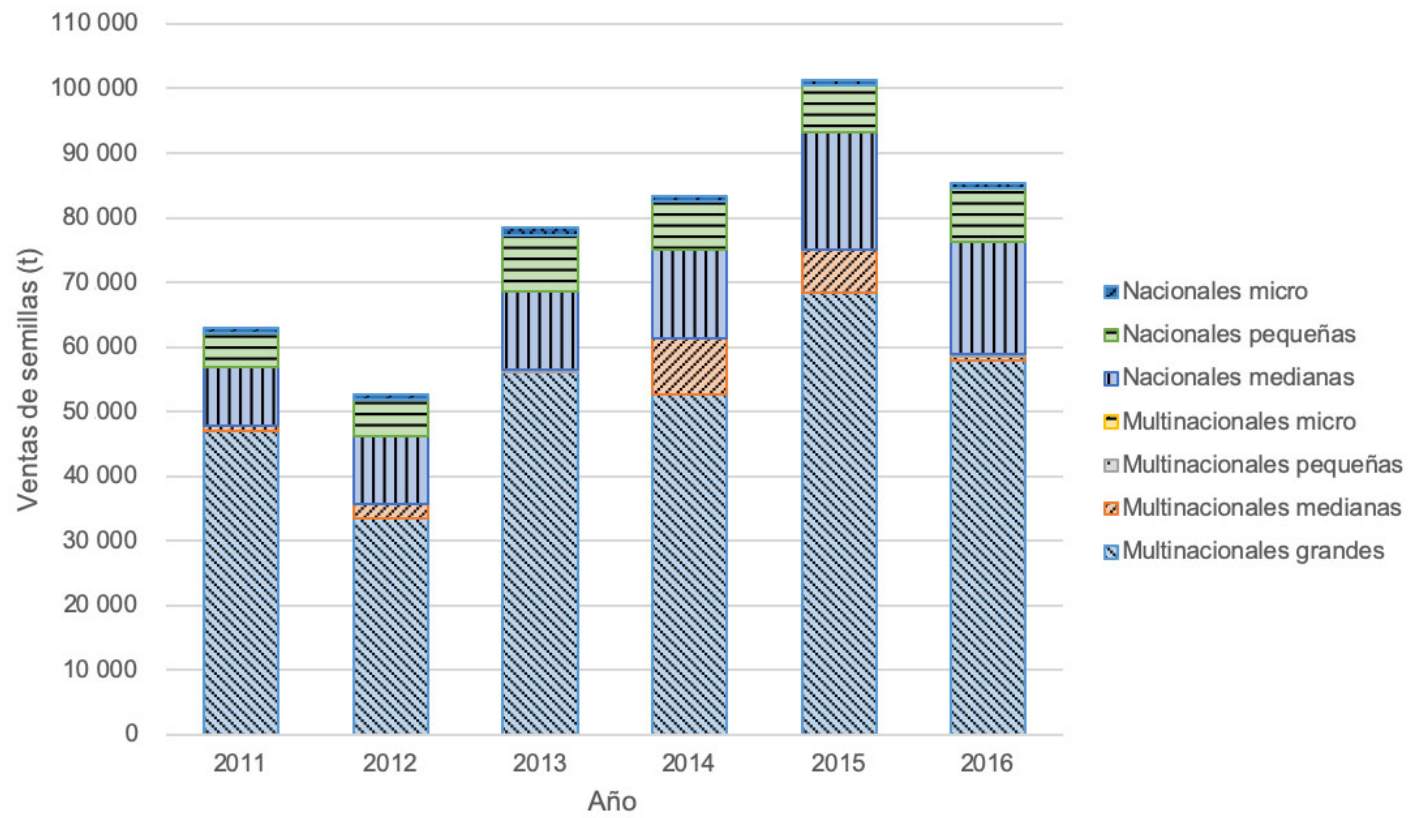

Figura 2. Ventas y participaciones de mercado del sector de semillas de maíz en México. 2011-2016.

Fuente: Datos de entrevistas y encuestas con empresas semilleras del consorcio MasAgro y de producción de semilla certificada del SNICS. Empresas grandes: $>5000$ t de semilla de maíz, medianas: 5000-500 t; pequeñas 500-50 t; micro $<50$ t.

Figure 2. Maize seed sales and market shares in Mexico. 2011-2016.

Source: Data from interviews and surveys of seed companies in the MasAgro consortium and SNICS certified seed production. Large companies: $>5,000$ t of maize seed, medium: 5,000-500 t; small 500-50 t; micro $<50 \mathrm{t}$. 
Las ventas del subsector nacional crecieron de 15000 a 27009 t de semillas de maíz, $75 \%$ entre 2011 y 2016 (Figura 3). El mayor crecimiento se dio en la categoría más grande (empresas medianas), que pasaron del 61,6\% al $66,6 \%$ de participación en ventas del subsector nacional en 2011 y 2016, respectivamente, con un promedio de 62,2 $\%$ en el periodo de estudio. A su vez, las empresas pequeñas y micro disminuyeron su participación, las primeras del $34,1 \%$ al $31,1 \%$, con un promedio de $33,5 \%$ y las segundas del $5,4 \%$ al 2,8 \%, con un promedio de 4,3\%

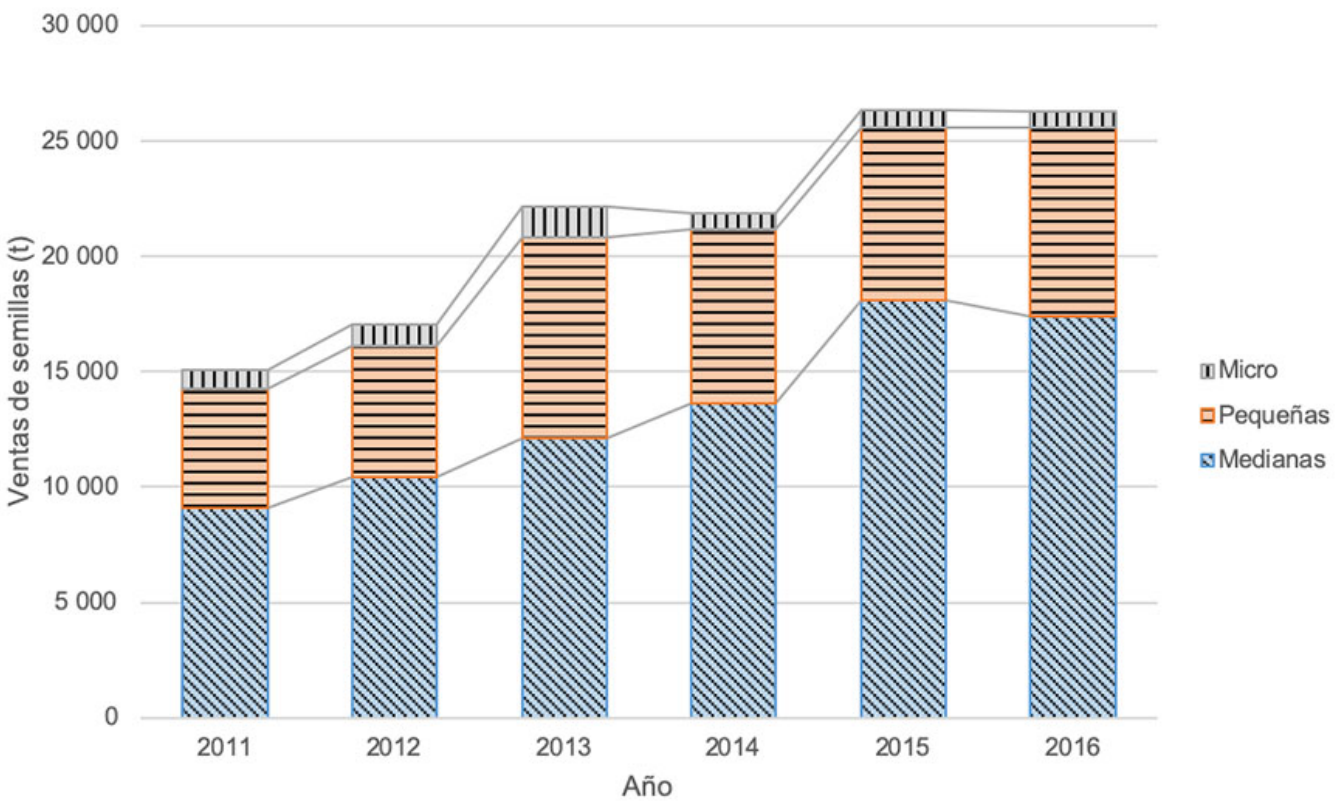

Figura 3. Ventas y participaciones de mercado del subsector nacional de semillas de maíz en México. 2011-2016.

Fuente: Datos de entrevistas y encuestas con empresas semilleras del consorcio MasAgro y de producción de semilla certificada del SNICS. Empresas grandes: >5000 t de semilla de maíz, medianas: 5000-500 t; pequeñas 500-50 t; micro <50 t.

Figure 3. Maize seed sales and market shares of the national sub-sector in Mexico. 2011-2016.

Source: Data from interviews and surveys of seed companies in the MasAgro consortium and SNICS certified seed production. Large companies: $>5,000 \mathrm{t}$ of maize seed, medium: 5,000-500 t; small 500-50 t; micro $<50 \mathrm{t}$.

Las empresas semilleras que participan en el consorcio de IMIC-LA representaron, en conjunto, el $85 \%$ de las ventas de empresas nacionales en 2016.

\section{Tipos de variedades}

De 85263 t de semilla de maíz producida y comercializada en 2016, el $90 \%$ fueron híbridos privados, 5 \% híbridos públicos, $2 \%$ variedades públicas y $3 \%$ fueron híbridos desarrollados con el consorcio IMIC-LA, correspondiente a la suma de $2 \%$ híbridos desarrollados por el CIMMYT y $1 \%$ por empresas privadas (Figura 4). Comparando con los datos disponibles que muestran los tipos de variedades en 1993, se observó el aumento de los híbridos privados y la disminución de híbridos y variedades públicos.

Al considerar el subsector nacional, los híbridos privados representaron las variedades más vendidas con el 69 $\%$ en 2016, seguidos por los híbridos públicos, nuevos híbridos desarrollados en CIMMYT durante el consorcio IMIC-LA, las variedades públicas y nuevos híbridos privados con germoplasma de IMIC-LA (Figura 5). En 


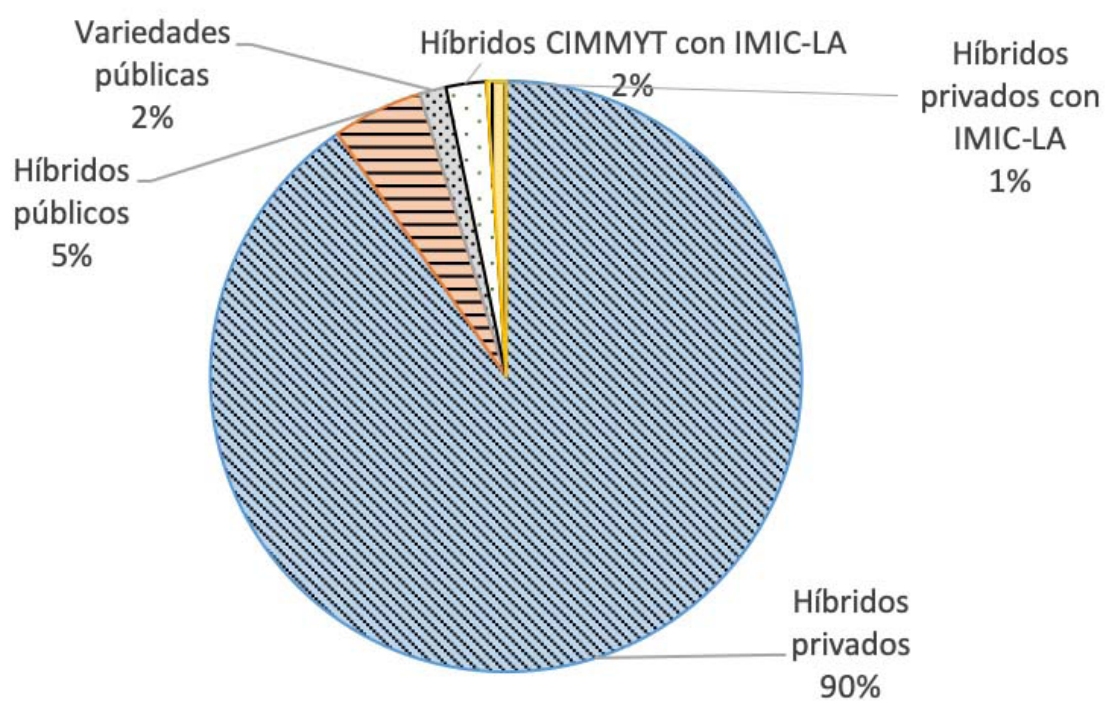

Figura 4. Híbridos y variedades de semillas de maíz de empresas nacionales y multinacionales en México. Se produjo un total de 85 $263 \mathrm{t}$ de semilla en el 2016.

Fuente: Datos de la encuesta de comercialización de semillas, 2016 y SNICS, 2016.

Figure 4. Hybrids and varieties of maize seeds from national and multinational companies in Mexico. Total of 85.263 tons in 2016.

Source: Data from the seed marketing survey, 2016 and SNICS, 2016.

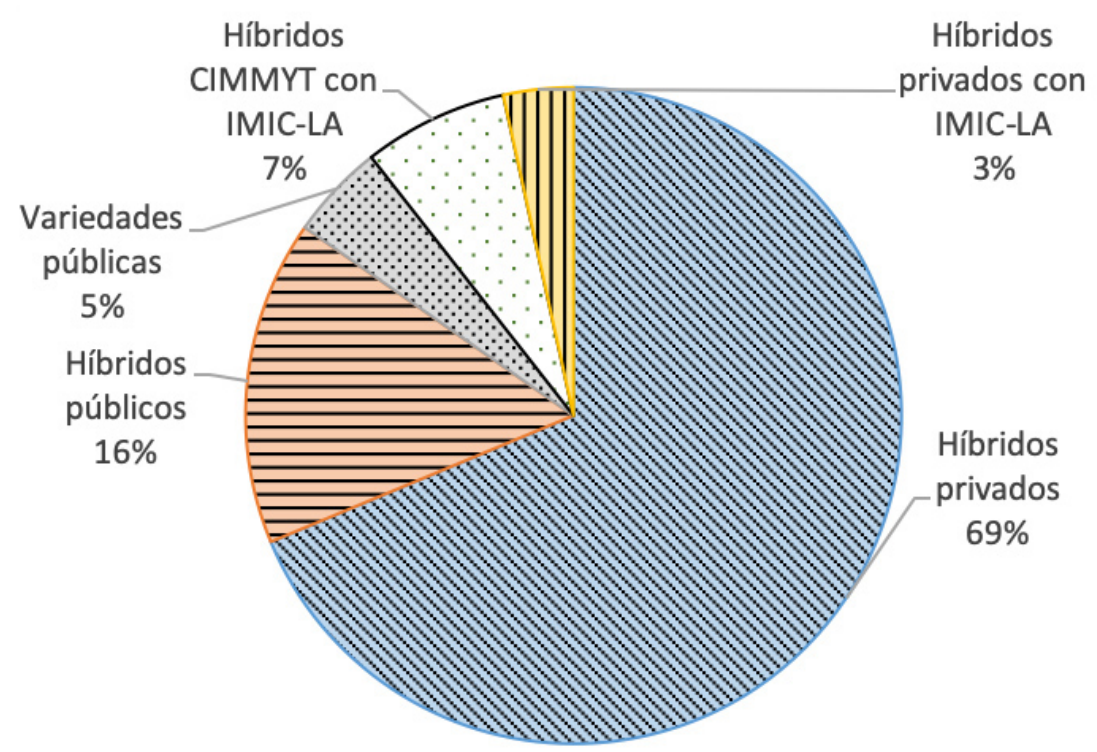

Figura 5. Tipos de variedades de semillas de maíz de empresas del subsector nacional en México. Total de 22269 t en 2016.

Fuente: datos de la encuesta de comercialización de semillas, 2016 y SNICS, 2016.

Figure 5. Types of maize seeds varieties in the national subsector in Mexico. Total of 22,269 tons in 2016.

Source: data from the seed marketing survey, 2016 and SNICS, 2016. 
total los híbridos desarrollados a través del consorcio IMIC-LA, es decir, ya sean cruzas finales desarrolladas en CIMMYT o cruzas finales desarrolladas por las empresas, representaron el $10 \%$ de las $22000 \mathrm{t}$ con datos. El total de ventas del sector semillero nacional, al sumar la producción de semillas certificadas reportadas por SNICS para otras empresas en 2016 y de las cuales no se tienen datos, fue de 26 mil toneladas. Las variedades de semillas desarrolladas con el consorcio representaron el $9 \%$ de la producción y ventas de todas las empresas del subsector nacional con datos en este estudio.

\section{Mercados}

Si se considera al mercado de semillas de maíz como toda la semilla sembrada por los agricultores, en México hay un mercado de aproximadamente 7,5 millones de hectáreas de las cuales 1,3 se cultivan bajo riego y 6,2 bajo temporal (SIAP, 2013a). Los híbridos de maíz se utilizan en prácticamente el $100 \%$ de las hectáreas cultivadas bajo riego. En el temporal, el promedio de uso es de $40 \%$ (SIAP, 2013b). La comparación con el promedio de uso de híbridos de $20 \%$ de la superficie de maíz (riego y temporal) 20 años atrás (datos de 1996), en una superficie similar, sugiere el crecimiento de la industria, sin considerar los cambios en el aumento de la densidad de siembra que significan mayor demanda de semilla. La cobertura con híbridos o penetración de mercado, presenta grandes diferencias según la región de producción (Cuadro 3). Occidente es el mercado de semillas más desarrollado en la producción de maíz bajo condiciones de temporal, seguido por el Bajío y la zona tropical de Occidente, donde la mayoría de los productores utilizan híbridos. El conjunto de regiones que incluyen el resto de las zonas tropicales, los valles altos y el subtrópico norte, tienen una cobertura intermedia entre 33 y $44 \%$, y los valles centrales de Oaxaca una cobertura del $12 \%$ de la superficie.

Cuadro 3. Cobertura con variedades de semillas mejoradas de maíz en las regiones de producción de temporal en México. 2013.

Table 3. Coverage with improved maize seed varieties in rainfed production regions in Mexico. 2013.

\begin{tabular}{lccc}
\hline Región & Cobertura con híbridos ( \%) & Superficie de maíz (ha) & Rendimiento de maíz (t/ha) \\
\hline Subtrópico Occidente & 90 & 617435 & 5,2 \\
Subtrópico Bajío & 68 & 442730 & 2,0 \\
Trópico Bajo Occidente & 66 & 481313 & 2,7 \\
Trópico Bajo Golfo & 44 & 783356 & 2,1 \\
Subtrópico Norte & 39 & 681.965 & 0,9 \\
Valles Altos México & 38 & 1203903 & 2,3 \\
Trópico Bajo Sur & 38 & 608739 & 1,9 \\
Trópico Bajo Península & 33 & 375121 & 1,4 \\
Valles Centrales Oaxaca & $y$ & 908459 & 1,5 \\
Sierras del Sureste & 12 & & 2,2 \\
Total & 38 & 6103021 & \\
\hline
\end{tabular}

Fuente: Elaboración de los autores con datos del SIAP 2013a y SIAP 2013b. Regiones de producción de temporal de Donnet et al. 2017 / Source: Authors' elaboration with data from SIAP 2013a and SIAP 2013b. Temporary production regions from Donnet et al. 2017. 


\section{Discusión}

Los resultados de la estructura mostraron un sector semillero sustancialmente más grande y competitivo después de 25 años de apertura y, especialmente, en los años más recientes. Además del crecimiento de las ventas de la industria en su conjunto, de 35000 en 1993 a 85000 en 2016, se destaca el rápido aumento del volumen y la participación de las ventas del subsector de empresas nacionales. La participación de mercado de estas empresas fue del $31 \%$ en 2016, notablemente más alta que el $5 \%$ registrado en 2009 , y resalta el desafío de mantener la posición sobresaliente alcanzada, y en lo posible aumentarla. El consorcio IMIC-LA puede desempeñar un papel principal en mantener y aumentar esta participación aprovechando y explotando las complementariedades de los socios públicos y privados. La discusión se centra en las implicaciones que los datos y análisis de la estructura pueden tener para informar la gestión de una asociación público-privada de semillas que ayude a mantener y mejorar esta participación y promover un sector semillero fuerte y competitivo.

Los centros de investigación públicos, tanto el CIMMYT como el INIFAP, tienen varias ventajas en el mejoramiento del maíz incluyendo una base sólida en instalaciones de investigación, bancos de germoplasma y conocimiento de la diversidad de materiales genéticos. En particular el CIMMYT resalta por su acceso a las herramientas y tecnologías de mejoramiento 'upstream' más modernas; algunas de estas tecnologías, como la edición génica, son particularmente promisorias para consorcios público-privados, especialmente en países en desarrollo, por su bajo costo y rápida producción (Friedrichs et al., 2019). El INIFAP, por su parte, ha lanzado más de doscientas variedades de maíz adecuadas para las diferentes regiones climáticas de México (Turrent-Fernández et al., 2014), y junto con otras instituciones nacionales como la Universidad Autónoma de Chapingo y el Colegio de Posgraduados, tiene el conocimiento territorial de las variedades de semilla. Las empresas aportan el conocimiento de los mercados, incluyendo las variedades preferidas por los agricultores y la capacidad de producción y distribución con los agricultores.

La principal ventaja de la colaboración en el consorcio es desarrollar la capacidad de prueba de las variedades en el campo. Dado que el rendimiento alcanzable de maíz está determinado por la interacción del germoplasma con el ambiente, la selección de las mejores variedades solo puede lograse a través de los ensayos en campo, lo cual está reflejado en que las grandes compañías de semillas de maíz emplean más agrónomos que mejoradores, y en las que un híbrido se puede probar en miles de ensayos de rendimiento en muchas condiciones antes de ser comercializado (Fischer et al., 2014). El consorcio ofrece esto a las empresas socias al posibilitar las pruebas que garanticen el rendimiento de las nuevas variedades en el campo de los agricultores donde se quieren vender las semillas.

La evidencia en este estudio acerca del aumento de las variedades de semillas híbridas versus las variedades de polinización abierta, y especialmente de los híbridos privados con respecto a los híbridos públicos, mostró lo que se ha llamado una proposición de valor ganar-ganar entre la industria de semilla y los agricultores (Fischer et al., 2014) y resalta, nuevamente, la importancia del acceso a los avances tecnológicos en mejoramiento y de desarrollar las pruebas a campo para identificar las variedades más competitivas. Como es ampliamente conocido, los híbridos fomentan la viabilidad de una industria comercial y un ambiente positivo para la inversión privada en el mejoramiento, porque la cruza de la primera generación (F1) presenta un mayor rendimiento por el vigor híbrido o heterosis que decae en la siguiente generación (F2), y por lo que los agricultores tienen el incentivo de comprar semilla todos los años.

Las nuevas semillas desarrolladas por los socios del consorcio también corresponden a solo este tipo de variedades. Los nuevos híbridos de maíz desarrollados, como buen indicador de la innovación del sector (Spielman y Kennedy, 2016), respaldan el papel del consorcio en posibilitar el aprovechamiento de los recursos de la investigación pública para entregar innovaciones de semilla a los agricultores. 
La orientación y foco de la colaboración público-privada de IMIC-LA en desarrollar variedades de maíz especialmente adaptadas a los ambientes de temporal en México, resalta aún más la competencia clave de las pruebas locales en la identificación y selección de las mejores variedades en ambientes donde la variabilidad ambiental hace que las mejores variedades cambien más de un lugar a otro. En total, las semillas mejoradas se siembran en aproximadamente 2,3 millones de hectáreas de maíz bajo temporal y existen 3,7 millones de hectáreas donde se podrían encontrar nuevos usuarios y clientes de híbridos. Como lo indican los rendimientos de maíz en el Cuadro 3, este potencial se encuentra en áreas cada vez más limitadas. Extender los beneficios de las semillas mejoradas a nuevos agricultores en estas áreas se relaciona con el reto en México de propiciar el uso extensivo de los materiales nativos sobresalientes a través de la producción y abastecimiento de semillas de variedades no convencionales (maíces nativos y combinaciones de semillas mejoradas y nativas acriolladas) (Ortiz et al., 2007; Espinosa et al., 2014). La diversidad de empresas semilleras y la participación de ambos el sector público con su interés en desarrollo y el sector privado con su interés comercial sugieren que el consorcio de semillas IMIC-LA puede tener una posición particularmente adecuada para abordar este reto.

La red de evaluación más que un activo complementario (Byerlee y Fischer, 2002) es un activo colaborativo y que puede constituir una ventaja competitiva para los socios del consorcio. Los resultados de este estudio sugieren que pueden existir oportunidades de aumentar la capacidad de la red de evaluación y que se pueden explorar en la gestión del consorcio. Por un lado, la falta de nuevas semillas que provengan de INIFAP entre los híbridos del consorcio, sugiere que la participación, con materiales y sitios de prueba de este socio está por debajo de su contribución potencial por sus capacidades y conocimientos del desempeño de germoplasma, incluido aquel que usan los agricultores y cuya incorporación podría mejorar el diseño y los resultados de las pruebas. Por su parte, la amplia participación de las empresas privadas en el consorcio sugiere un potencial para aumentar el número de sitios y materiales que se ensayan y que se traducirían en un aumento de nuevas variedades de semillas comercializadas en los próximos años.

El intercambio de los dos tipos de materiales de mejoramiento, cruzas finales y líneas y cruzas simples intermedias, parece apropiado dados los distintos tipos de empresas, con y sin programas de mejoramiento, y su interés en producir híbridos privados. La posibilidad de utilizar cruzas finales sugiere un aprovechamiento de las complementariedades, lo que reduciría la competencia entre los sectores público y privado en el caso de las empresas sin capacidad de mejoramiento, mientras el acceso a las cruzas y líneas intermedias permite la complementariedad con las empresas más grandes y que dependen del germoplasma desarrollado en estas organizaciones para generar sus propias variedades de semillas comerciales (híbridos propietarios).

Con respecto a los socios nacionales del sector público, una preocupación persistente es que las asociaciones público privadas puedan suplantar su papel de los socios nacionales en la investigación agrícola (Spielman et al., 2007). Todo esto significa que hay una necesidad de desarrollar los acuerdos de transferencia e intercambio de materiales genéticos y de la información acerca del desempeño de las variedades de semilla. Dado que los beneficios de la cooperación y la colaboración dependen significativamente de la capacidad de los actores públicos y privados para desarrollar arreglos creativos para reducir la principal fuente de competencia entre sectores que es la propiedad y el uso de conocimiento científico y tecnológico (Spielman y von-Grebmer, 2004), el consorcio tiene que trabajar en estas capacidades.

La información en este estudio sobre el número de las empresas, los tipos de variedades y volumen de ventas se puede utilizar como punto de partida para desarrollar y negociar "acuerdos y mecanismos creativos" (ibid) entre los miembros del consorcio sobre los términos y condiciones del intercambio de materiales e información para el uso de la propiedad intelectual que aumenten el uso e impacto / beneficios de los productos de mejoramiento del consorcio. La cantidad y diversidad de empresas (y cobertura geográfica derivada), sugiere que estos arreglos pueden implicar un "reacomodamiento" y avance hacia arreglos entre las empresas de especialización, por ejemplo, 
producir material parental, cruzas y líneas intermedias de semilla básica, y producir híbridos finales, es decir, empresas multiplicadoras.

\section{Conclusiones}

Este estudio presenta para el sector de semillas de maíz, una actualización de los datos e indicadores de tamaño, empresas, tipos de variedades y cobertura de mercados, incluyendo la participación del consorcio público privado IMIC-LA, como nueva forma de coordinación entre la investigación y desarrollo, y la producción y comercialización de semillas. Los datos disponibles con el consorcio de semillas IMIC-LA han proporcionado la oportunidad de actualizar la evidencia y proveer información detallada para profundizar en un sector de gran interés para México. Esta información, que no está comúnmente accesible dado que involucra información privada de las empresas, se generó por la necesidad de demostrar el impacto de la utilización de fondos públicos en el mejoramiento de maíz.

El CIMMYT y los institutos públicos de investigación de México han sido la fuente de las variedades mejoradas e híbridos de maíz, multiplicados y distribuidas por las empresas nacionales, así como de las líneas y cruzas utilizadas como materiales de mejoramiento. La forma de la coordinación era mediante el abastecimiento de semilla básica y no había un intercambio sistemático de información sobre el origen y el uso y desempeño del germoplasma. Lo que es diferente con el consorcio es que involucra pruebas colaborativas de los materiales y un mayor intercambio de la información, no solo entre una empresa y un centro de mejoramiento sino entre todas las empresas y centros de mejoramiento miembros.

La amplia participación de las empresas como miembros del consorcio y el desarrollo y comercialización de nuevas variedades de semillas sugieren un impacto positivo y un papel en el crecimiento y competitividad de las empresas semilleras nacionales. La contribución de la alianza público privada al crecimiento del subsector nacional es particularmente significativa en el contexto de competencia con empresas multinacionales líderes y provee un modelo alternativo exitoso de coordinación de la cadena de producción de semillas. En una industria más madura y más competitiva, el rol del consorcio es aumentar la información que posibilite a las empresas el acceso a germoplasma y la identificación de las mejores variedades de semillas en las condiciones específicas de los agricultores a los que se intenta vender, y a los centros de mejoramiento, la demostración de los beneficios de las semillas en los agricultores a los que se intenta impactar.

El principal activo y actividad del consorcio es el intercambio de información en la red colaborativa de evaluación que permite identificar los híbridos de mejor y más estable desempeño, y las variaciones entre una zona y otra. Esta información es útil para las empresas para planificar la producción, distribución y promoción de las semillas. También es útil para los mejoradores que buscan identificar nuevas prioridades y necesidades de mejoramiento.

La iniciativa de IMIC-LA es un ejemplo de esfuerzos intensivos en la recopilación y análisis de datos que son relevantes para mejorar la gestión del consorcio y validar su impacto, y para entender los sistemas de semillas en general. A nivel de la gestión del consorcio, puede ser útil para construir indicadores de participación de los socios, diseñar planes de mercado en áreas específicas y con agricultores específicos, incluyendo qué tipos de acuerdos promover con qué tipos de empresas y en qué términos de cantidad y tipos de variedades de semilla.

A nivel de validar el impacto, se puede combinar y triangular con información del uso de las semillas por los agricultores y las diferencias de estas semillas en rendimiento, calidad para distintos usos y rentabilidad con respecto a otras opciones de semilla. El fortalecimiento del sector semillero se tiene que verificar finalmente en el aumento de la productividad agrícola. Es importante avanzar en la evaluación de cómo las semillas ayudan a los agricultores, e integrar los indicadores de la estructura del sector semillero con indicadores del desempeño 
en el campo e indicadores de desarrollo. En general, la disponibilidad de estos datos para los investigadores y los tomadores de decisiones en los sectores público, privado y de la sociedad civil, es importante para informar mejor los debates públicos y la formulación de políticas de semillas que, junto con prácticas de producción y comercialización, resulten en un aumento de la producción y sustentabilidad del maíz.

\section{Agradecimientos}

Los autores agradecen el apoyo financiero de la Secretaría de Agricultura, Ganadería, Desarrollo Rural, Pesca y Alimentación (SAGARPA), del Gobierno de México a través de la iniciativa de Modernización Sustentable de la Agricultura Tradicional (MasAgro). Adicionalmente, se agradecen los comentarios de dos revisores anónimos a una versión anterior de este manuscrito y que ayudadaron a mejorar este trabajo.

\section{Literatura citada}

Abate, T., B. Shiferaw, A. Menkir, D. Wegary, Y. Kebede, K. Tesfaye, M. Kassie, G. Bogale, B. Tadesse, and T. Keno. 2015. Factors that transformed maize productivity in Ethiopia. Food Secur. 7:965-981. doi:10.1007/s12571-015-0488-Z

Beintema, N., G.J. Stads, K. Fuglie, and P. Heisey. 2012. ASTI global assessment of agricultural r\&d spending: developing countries accelerate investment. IFPRI, Washington, USA.

Byerlee, D., and K. Fischer. 2002. Accessing modern science: Policy and institutional options for agricultural biotechnology in developing countries. World Dev. 30:931-948. doi:10.1016/S0305-750X(02)00013-X

Castañeda, Y., A. González, M. Chauvet, y J.F. Ávila. 2014. Industria semillera de maíz en Jalisco: Actores sociales en conflicto. Sociology 29(83):241-278.

Chisinga, B. 2011. Seeds and subsidies: The political economy of input programmes in Malawi. IDS Bull. 42(4):59-68.

Donnet, M.L., I.D. López, J.R. Black, and J. Hellin. 2017. Productivity differences and food security: a metafrontier analysis of rain-fed maize farmers in MasAgro in Mexico. AIMS Agric. Food 2:129-148. doi:10.3934/agrfood.2017.2.129

Escandón, D., G. Sosa, y M.C. Castro. 2017. MasAgro, desarrollo y producción de semilla de maíz de calidad con herramientas moleculares. MasAgro, MEX. http://www.masagro.org/es/rss-conservacion/2291-masagro-desarrollo-y-produccion-desemilla-de-maiz-de-calidad-con-herramientas-moleculares (consultado 10 set. 19).

Espinosa, A., M. Sierra, y N. Gómez. 2003. Producción y tecnología de semillas mejoradas de maíz por el INIFAP en el escenario sin la PRONASE. Agron. Mesoam. 14:117-121. doi:10.15517/am.v14i1.11998

Espinosa, A., A. Turrent, M. Tadeo, A. San-Vicente, N. Gómez, R. Valdivia, M. Sierra, y B. Zamudio. 2014. Ley de semillas y ley federal de variedades vegetales y transgénicos de maíz en México. Rev. Mex. Cienc. Agríc. 5:293-308. doi:10.29312/ remexca.v5i2.967

Flaherty, K., G.J. Stads, and A. Srinivasacharyulu. 2013. benchmarking agricultural research indicators across asia-pacific. IFPRI, WA, USA.

Fischer, R., D. Byerlee, and G. Edmeades. 2014. Crop yields and global food security: will yield increase continue to feed the world? ACIAR Monographics, Canberra, AUS.

Friedrichs, S., Y. Takasu, P. Kearns, B. Dagallier, R. Oshima, J. Schofield, and C. Moreddu. 2019. Meeting report of the OECD conference on "Genome editing: Applications in agriculture-implications for health, environment and regulation". Transgenic Res. 28:419-463. doi:10.1007/s11248-019-00154-1 
García, J.A., y R. Ramírez. 2014. El mercado de la semilla mejorada de maíz (Zea mays L.) en México: análisis del saldo comercial por entidad federativa. Rev. Fitotec. Mex. 37(1):69-77.

González, A., J. Islas, A. Espinosa, J.A. Vázquez, y S. Wood. 2008. Impacto económico del mejoramiento genético del maíz en México. INIFAP, Ciudad de México, MEX.

Hamukwala, P., G. Tembo, J. Mark Erbaugh, and W. Donald Larson. 2012. Improved seed variety value chains in Zambia: A missed opportunity to improve smallholder productivity. Afr. J. Agric. Res. 7:4803-4818. doi:10.5897/AJAR12.527

Hellin, J., and C. Camacho. 2017. Agricultural research organisations' role in the emergence of agricultural innovation systems. Dev. Prac. 27:111-115. doi:10.1080/09614524.2017.1256373

Kassie, G.T., O. Erenstein, W. Mwangi, J. MacRobert, P. Setimela, and B. Shiferaw. 2013. Political and economic features of the maize seed industry in Southern Africa. Agrekon 52:104-127. doi:10.1080/03031853.2013.798067

Langyintuo, A.S., W.M. Mwangi, A.O. Diallo, J. MacRobert, J. Dixon, and M. Banziger. 2010. Challenges of the maize seed industry in eastern and southern Africa: A compelling case for private-public intervention to promote growth. Food Policy 35:323-331. doi:10.1016/j.foodpol.2010.01.005

López, M.A. 1995. Las industrias de semilla de maíz de Centro América y México: relaciones entre los sectores público y privado. Agron. Mesoam. 6:157-168. doi:10.15517/am.v6i0.24824

Luna, M.B., A. Hinojosa, O. Ayala, F. Castillo, y J. Mejía. 2012. Perspectivas de desarrollo de la industria semillera en México. Rev. Fitotec. Mex. 35(1):1-7.

Mabaya, E. 2016. Performance of the formal seed sector in Africa: Findings from the African seed access index. Presented at: Fifth International Conference, Addis Ababa, ETH. September 23-26.

MacRobert, J.F. 2014. Administración de empresas productoras de semilla en África. Trad. Alma McNab. CIMMYT, MEX.

Morris, M.L., and M.A. López. 1999. impacts of maize breeding research in Latin America, 1966-1997. CIMMYT, México D.F., MEX.

Napasintuwong, O. 2014. Seed industry in Thailand: Market structure, regulations, policies and prospect. Presented at: 18th International Consortium on Applied Bioeconomy Research: Bioeconomy and development, Nairobi, KEN. June 18-20.

Ortiz, C., P. Ortega, G. Molina, R. Mendoza, C. Mendoza, G. Castillo, O. Muñoz, A. Turrent, y Y. Kato. 2007. Análisis de la problemática de la producción nacional de maíz y propuesta de acción. Universidad Aautónoma de Chihuahua, Colegio de Postgraduados, e Instituto Nacional de Investigaciones Forestales, Agrícolas y Pecuarias, Chapingo, MEX.

Prasanna, BM. 2013. Maize in the developing world: trends, challenges, and opportunities. Indonesian Center for Food Crop research and Development (ICFORD), IND. http://pangan.litbang.pertanian.go.id/files/IMC-PDF/03-Prasanna.pdf (accessed Dec. 4, 19).

Pray, C., and L. Nagarajan. 2013. Role of biotechnology in stimulating agribusiness R\&D investment in India. J. Agrobiotechnol. Manag. Econ. 16(2):104-111.

Pray, C., and L. Nagarajan. 2012. Innovation and research by private agribusiness in India. IIFPRI, WA, USA.

Ragonnaud, G. 2013. The EU seed and plant reproductive material market in perspective: a focus on companies and market shares. European Union, Brussels, BEL.

Schenkelaars, P., H. de-Vriend, and N. Kalaitzandonakes. 2011. Drivers of consolidation in the seed industry and its consequences for innovation commission on genetic modification. LIS CONSULT, Utrecht, NLD. https://www.lisconsult. nl/files/docs/consolidation_seed_industry.pdf (accessed Oct. 17, 2018). 
SIAP (Sistema de Información Agrícola y Pecuaria). 2013a. Anuario estadístico de la producción agrícola. SIAP, MEX. http:// infosiap.siap.gob.mx/aagricola_siap/icultivo/index.jsp (consultado 5 may. 2018).

SIAP (Sistema de Información Agrícola y Pecuaria). 2013b. Estadística de uso tecnológico y de servicios en la superficie agrícola. SIAP, MEX. https://www.gob.mx/siap/documentos/tecnificacion (consultado 5 may. 2018).

Sisay, D.T., F.J.H.M. Verhees, and H.C.M. van-Trijp. 2017. Seed producer cooperatives in the Ethiopian seed sector and their role in seed supply improvement: A review. J. Crop Improv. 3:323-355. doi:10.1080/15427528.2017.1303800

Smale, M., and J. Olwande. 2014. Demand for maize hybrids and hybrid change on smallholder farms in Kenya. Agric. Econ. 45:409-420. doi:10.1111/agec.12095

SNICS (Servicio Nacional de Inspección y Certificación de Semillas). 2016. Producción de semilla certificada de maíz ciclos 2011/2016. SNICS, Tlalnepantla, MEX.

Spielman, D., and K. von-Grebmer. 2004. Public-private partnerships in agricultural research: An analysis of challenges facing industry and the Consultative Group on International Agricultural Research. EPTD discussion papers 113. International Food Policy Research Institute (IFPRI), WA, USA.

Spielman, D., F. Hartwich, and K. von-Grebmer. 2007. Sharing science, building bridges, and enhancing impact: public-private partnerships in the CGIAR. IFPRI, WA, USA.

Spielman, D., and A. Kennedy. 2016. Towards better metrics and policymaking for seed system development: Insights from Asia's seed industry. Agric. Syst. 147:111-122. doi:10.1016/j.agsy.2016.05.015.

Spielman, D., D. Kolady, P. Ward, H. Ar-Rashid, and K. Gulati. 2012. Public expenditures, private incentives, and technology adoption: the economics of hybrid rice in south Asia. IFPRI, WA, USA.

Tripp, R., and A. Mensah-Bonsu. 2013. Ghana's commercial seed sector: new incentives or continued complacency? IFPRI, WA, USA.

Tripp, R., and C. Ragasa. 2015. Hybrid maize seed supply in Ghana. IFPRI, WA, USA.

Turrent, A., A. Espinosa, J. Cortés, y H. Mejía Andrade. 2014. Análisis de la estrategia MasAgro-maíz. Rev. Mex. Cienc. Agríc. 8:1531-1547. https://doi.org/10.29312/remexca.v5i8.833

Turrent, A, J.I. Cortés, A. Espinosa, E. Hernández, R. Camasz, J.P. Torres, y A. Zambada. 2017. MasAgro o MIAF ¿Cuál es la opción para modernizar sustentablemente la agricultura tradicional de México? Rev. Mex. Cienc. Agríc. 8:1169-1185. doi:10.29312/remexca.v8i5.116 